\title{
LAS PERIODISTAS Y TRABAJADORAS DE LA COMUNICACIÓN FRENTE A LAS VIOLENCIAS Y DISCRIMINACIÓN EN MEDIOS TRADICIONALES EN ECUADOR
}

\section{WOMEN JOURNALISTS AND COMMUNICATION WORKERS FACING VIOLENCE AND DISCRIMINATION IN TRADITIONAL MEDIA IN ECUADOR}

Michelle Moretti

Consejo de Comunicación, Ecuador

(D) https://orcid.org/0000-0002-9409-6634

Autor para correspondencia: Michelle Moretti, email: michellemoretti@hotmail.com

\section{Resumen}

El presente documento analiza la cultura periodística para ofrecer un panorama general de la situación de las periodistas y trabajadoras de la comunicación en radio, televisión y medios impresos, desde una mirada universal hasta elementos propios del caso ecuatoriano. A través de una revisión del marco legal y teórico, junto a elementos históricos y cifras, como el Registro Público de Medios y el Monitoreo de Contenidos del Consejo de Comunicación, se presentarán datos sobre la realidad de las mujeres que laboran en la esfera mediática. Se expondrán algunas prácticas de violencias y discriminación contra las periodistas, ligadas a temas como los llamados techo de cristal y suelo pegajoso, para reflexionar sobre actitudes recurrentes que atentan contra su seguridad, integridad y progreso tanto al interior de medios tradicionales, como en lo público y su relación con las audiencias. Además, se ofrecerán algunas pautas técnicas para superar esta problemática, que afecta tanto al ser humano en su calidad de mujer, como a la libertad de expresión y de prensa.

Palabras clave: mujeres, periodismo, medios de comunicación, cultura periodística.

\begin{abstract}
This document analyzes journalistic culture to offer a general panorama of the situation of journalists and communication workers in radio, television and printed media, from a universal perspective to elements regarding the Ecuadorian case. Through a review of the legal and theoretical framework, together with historical elements and figures, such as the Public Media Registry and the Monitoring Content of the Consejo de Comunicación, data on the reality of women who work in the media sphere will be presented. Some practices of violence and discrimination against journalists will be exposed, linked to issues such as the so-called glass ceiling and sticky
\end{abstract}

Global Media Journal México, 18(34), 94-110, enero - junio 2021. 
floor, to reflect on recurring attitudes that threaten their safety, integrity and progress both within traditional and public media, and its relationship with audiences. In addition, some technical guidelines will be offered to overcome this issue, which affects both the human being as a woman, as well as freedom of expression and press.

Keywords: women, journalism, mass media, journalistic culture.

Recibido: 02/03/2021

Aceptado: 05/07/2021

\section{Introducción}

Los medios de comunicación son actores sociales que, como cualquier institución, tienden a reflejar los valores del entorno donde funcionan; $y$, al igual que en muchos países, en Ecuador está presente una cultura que privilegia a los hombres, como lo evidencian varias investigaciones que se expondrán a lo largo de este documento. Por su parte, las mujeres involucradas en estos espacios enfrentan desafíos más allá de sus tareas, como resultado de procesos históricos trasversales de violencias y discriminación relacionadas al género.

Para entender esta dinámica, se hará una aproximación a la construcción estructural de la cultura mediática tradicional ecuatoriana, como base para ubicar algunos elementos recurrentes que afectan la participación de las mujeres en los distintos niveles de responsabilidad, como invisibilización y subrepresentación. Paralelamente, el análisis de textos jurídicos y teóricos servirá para entender, de forma legal e integral, los riesgos, obstáculos y avances presentes en su progreso laboral, con especial atención en las periodistas, por ser quienes tienen mayor exposición al público.

Se utilizará el análisis de cifras, tales como las del Registro Público de Medios y el Monitoreo de Contenidos del Consejo de Comunicación, como herramienta para cuantificar la presencia de las mujeres en radio, televisión y medios impresos; al tiempo que, se reflexiona sobre los desafíos en temas de derechos, que inciden tanto en la persona por ser mujer, como en la libertad de expresión y de prensa.

\section{Marco teórico}

\section{Aproximación a la cultura periodística de Ecuador}

Al ser la cultura uno de los principales marcos del análisis propuesto, se partirá de definir las ideas que la acompañan; en ese sentido, será entendida, según Mead (1982), como "el conjunto de formas adquiridas de comportamiento, formas que ponen de manifiesto juicios de valor sobre las condiciones de vida, que un grupo humano de tradición común

Global Media Journal México, 18(34), 94-110, enero - junio 2021. 
transmite mediante procedimientos simbólicos, de generación en generación" (p. 36-37). Lejos de describir a un pueblo o nacionalidad milenaria, será utilizado para enmarcar el conjunto de prácticas sociales que se dan en un determinado momento temporal y espacial.

Bajo esa línea, la noción de cultura periodística comprende las ideas culturales que el trabajador del ámbito comunicacional usa tanto para evaluar y ordenar su realidad, como en su participación como gremio de manera universal, diversa y heterogénea (Hanitzsch, 2007; Oller y Chavero, 2018). En la práctica, en países como Ecuador está presente en la “abrumadora composición masculina de la profesión [que] ha desembocado en los valores masculinos" (Proyecto de Monitoreo Global de Medios, 2015a, p. 49), con la prevalencia de ciertos roles donde los hombres ocupan la mayor parte de los puestos de prestigio y poder (Oller et al., 2016; Ross et al., 2016); esto convierte a los medios de comunicación en espacios difíciles para la incursión de las mujeres.

A continuación se analizarán algunos puntos de la historia del periodismo ecuatoriano que evidencian esta realidad, junto a hechos recurrentes a nivel mundial. Para ello, se retrocederá hasta antes del establecimiento de la República, en la persona de Manuela Espejo.

En gran medida su figura quedó en la penumbra, salvo por un elemento clave, como el que dejó esculpido en los primeros números, del primer periódico de la Audiencia: Primicias de la Cultura. [...]
"Erophilia”, seudónimo que utilizó quien fuera la primera mujer que se atrevió a expresar sus criterios a través de la prensa (Paladines, 2004, p. 8).

La clandestinidad a la que fue sometida Espejo, y otras mujeres, no fue una actitud superada en el Ecuador ya constituido pues, por mucho tiempo, la escritura femenina era mal vista al ser asumida como "una actividad que desvirtúa sus naturales deberes y atenta contra la santidad conyugal" (Rodas, 2011, p. 33). No fue hasta 1887 que algunas mujeres enfrentaron abiertamente esa tradición para ejercer el periodismo.

Como lo indica Calvopiña (2019), “entre las pioneras está Zoila Ugarte, quien bajo el nombre de Zarelia, publica lo que supone son las primeras obras periodísticas escritas por una mujer en Ecuador" (párr. 3). Esta militante feminista trabajó en el semanario Tesoro del Hogar entre 1887 y 1893; en 1905, con su nombre de pila, participó de la que se cataloga como la primera revista del país escrita por ellas, “La Mujer" (Rodas, 2011); y, para 1911, fue la primera directora y redactora del periódico político La Prensa (Casa de la Cultura Ecuatoriana, s.f.).

Lo excepcional de Espejo y Ugarte es que, inscritas en sus propias coyunturas, lograron sobrepasar un proceso estructural histórico que se conoce como aniquilación simbólica: describe la omisión, banalización y condena de las mujeres en los medios de comunicación tradicionales, mediante su ausencia o subrepresentación (Tuchman et al., 1978), el último término coligado a encasillarlas a

Global Media Journal México, 18(34), 94-110, enero - junio 2021. 
ciertos oficios de menor rango, con poco o nulo poder de decisión.

Dicho proceso subsiste en los inicios de la profesionalización del periodismo ecuatoriano en los 40's. Como lo señala Plaza (2014), en la creación de la Escuela de Comunicación Social de la Universidad Central del Ecuador y la Escuela de Información de la Universidad Estatal de Guayaquil, auspiciadas por directivos de El Comercio y El Universo, se perfila la figura de los hombres como eje central del funcionamiento de los medios. Como consecuencia, las mujeres publican pocos artículos de prensa y los datos que existen sobre sus contribuciones están dispersos mayormente en memoria oral; así también, sus primeras incursiones en televisión fueron mediadas por estereotipos de belleza o domésticos.

Ya en épocas recientes y hasta la actualidad, la anulación y subrepresentación continúa porque, como manifiestan la Declaración y Plataforma de Acción de Beijing (ONU Mujeres, 1995), la UNESCO (2014), la ONU Mujeres (2020), y el Proyecto de Monitoreo Global de Medios (2015b), junto a varias organizaciones y documentos, son pocas las mujeres que llegan a ocupar cargos altos o formar parte de juntas directivas y órganos que influyen en la política de los medios de difusión ya que estos roles siguen siendo reservados para los hombres; aunque, cabe reconocer que, gracias al acceso a la profesionalización aumentó el número de mujeres (Beate et al., 2019). La realidad actual, mundial y nacional, muestra que las dificultades que enfrentan las mujeres están más allá del ingreso a los medios y tienen que ver con su capacidad de progresar en su carrera (UNESCO y Universidad de Oxford, 2018).

Según The Global Report on the Status of Women in the News Media, "las mujeres representan solo un tercio de la fuerza laboral del periodismo" (International Women's Media Foundation [IWMF], 2011, p. 6), dato que parte de lo universal y se aplica a Ecuador. Por mencionar unas cifras, en la Carrera de Comunicación de la Universidad Central del Ecuador (2009-2020), en los últimos 20 años, las mujeres superan a los hombres en matriculación; sin embargo, ciertas áreas de los medios de comunicación mantienen un esquema de roles, como lo evidencia el mencionado Reporte Global (IWMF, 2011): en el país las mujeres ocupan el 20,8\% de cargos gobernancia y toma de desiciones; el 40,7\% de puestos de alta jerarquía; $27.5 \%$ de cargos gerenciales; $32,5 \%$ de cargos de dirección; y, 45.4\% son profesionales calificadas como periodistas.

Se puede decir que, pese a las incesantes luchas de las mujeres por sus derechos, aún está vigente una estructura histórica constituida para y por hombres como base de la cultura periodística universal que abarca el caso ecuatoriano; y que, además, presenta desigualdades de género (Byerly y Ross citadas por Consejo de Comunicación, 2017). Frente a esta realidad, existen varios instrumentos legales que ponen en la palestra las violencias y discriminación contra las mujeres en lo laboral y, como se verá a continuación, existen especificidades para las instituciones mediáticas. 


\section{Las periodistas y las trabajadoras de la comunicación en el marco del derecho}

Si bien instrumentos legales de alta jerarquía, informes y pronunciamientos oficiales serán claves para esta reflexión, se cree oportuno señalar ciertos extractos de documentos para socializar algunos avances en la materia, que se aplican a Ecuador.

De manera general rigen dogmas sobre mujeres y trabajo. En la Constitución de la República del Ecuador (2008), a lo largo de su contenido, prohíbe la discriminación y violencias; por ejemplo, en el Artículo 331 dice:

El Estado garantizará a las mujeres igualdad en el acceso al empleo, a la formación y promoción laboral $y$ profesional, a la remuneración equitativa, y a la iniciativa de trabajo autónomo. Se adoptarán todas las medidas necesarias para eliminar las desigualdades.

Se prohibe toda forma de discriminación, acoso o acto de violencia de cualquier indole, sea directa o indirecta, que afecte a las mujeres en el trabajo.

Así también, existen textos que buscan salvaguardar la integridad de los periodistas y trabajadores de la comunicación en universal. Están, por ejemplo, la Declaración de Chapultepec (2019); o, la inserción de este particular, en el artículo 42.1, en la Ley Orgánica de Comunicación reformada (2019). Esta última, contempla la obligatoriedad de una nómina con equidad y paridad entre hombres y mujeres para los medios de carácter nacional.

Las violencias y discriminaciones contra las periodistas y trabajadoras de la comunicación están descritas en documentos como el Informe de la Relatoría Especial para la Libertad de Expresión de la CIDH sobre Mujeres periodistas y libertad de expresión (Organización de los Estados Americanos [OEA], 2018) que, en uno de sus párrafos señala:

7. Tanto las mujeres periodistas como aquellas que trabajan en diferentes áreas de la comunicación deben lidiar con entornos amenazantes especificos que restringen su labor e impactan desproporcionadamente en el ejercicio de su derecho a la libertad de expresión. Desde la desigualdad en el ámbito laboral, los comentarios sexistas y misóginos, la violencia sexual o asesinatos de mujeres en razón de su género (o feminicidios), las amenazas o riesgos que enfrentan las mujeres periodistas tienden a ser invisibilizados y no son reconocidos como restricciones indebidas a la libertad de expresión por la mayoría de los colegas, medios de comunicación y autoridades estatales (2018, p. 13).

Para enriquecer el debate y aprovechar el contenido legal, junto algunos aportes teóricos e investigativos, se procederá a reflexionar sobre las violencias y discriminación contra las periodistas, por ser quienes están en el medio como trabajadoras y a la vista del público.

Global Media Journal México, 18(34), 94-110, enero - junio 2021. 


\section{Violencias y discriminación contra las periodistas de medios de comunicación}

Si bien todas las trabajadoras de la comunicación están expuestas a violencias y discriminación por temas de género, el análisis se especializará en el periodismo. Este abordaje busca denotar la segregación que afrontan las profesionales al interior de los medios y las afectaciones resultantes de su exposición pública porque atentan contra la persona y los derechos conexos a la comunicación. Y es que...

[...] es particularmente grave en el caso de mujeres que ejercen la libertad de expresión de manera activa y mantienen un alto perfil público, tales como las mujeres periodistas [...].Al desafiar estereotipos machistas que reprueban su participación en la vida pública, se ven enfrentadas a la violencia y la discriminación contra las mujeres basada en su género, así como a formas diferenciadas de violencia por parte de actores estatales y no estatales. A su vez, enfrentan desprotección y obstáculos en el acceso a la justicia, también diferenciados de sus compañeros varones (OEA, 2018, p. 11-12).

Para entender cómo se presentan las violencias y discriminación, se iniciará con su conceptualización. La Ley para Prevenir y Erradicar la Violencia contra las Mujeres (2018), señala que "la violencia de género contra las mujeres [es] cualquier acción o conducta basada en su género que cause o no muerte, daño y/o sufrimiento físico, sexual, psicológico, económico o patrimonial, gineco-obstétrico a las mujeres, tanto en el ámbito público como privado" (Art. 4, Num. 1). La mencionada Ley describe varios tipos de violencia (Art. 10), siendo la física, psicológica, sexual, simbólica, económica y patrimonial aquellas que les pueden ocurrir en el ámbito laboral (Art. 12, Num. 3) a las periodistas.

Sobre la discriminación contra la mujer, ésta es...

[...] toda distinción, exclusión o restricción basada en el sexo que tenga por objeto o resultado menoscabar o anular el reconocimiento, goce o ejercicio por la mujer, independientemente de su estado civil, sobre la base de la igualdad del hombre y la mujer, de los derechos humanos y las libertades fundamentales en las esferas politica, económica, social, cultural y civil o en cualquier otra esfera. (Convención sobre la Eliminación de Todas las Formas de Discriminación contra la Mujer, 1979, Art.1)

Ahora bien, antes de abordar las violencias que enfrentan las mujeres que ejercen el periodismo, es oportuno aclarar que este análisis: uno, no excluye a los hombres como posibles víctimas de los peligros intrínsecos en la profesión, sino que particulariza acciones que van más allá; dos, se ha mantenido un discurso que habla de hombres y mujeres, pero no se desconoce la existencia de la diversidad de géneros

Global Media Journal México, 18(34), 94-110, enero - junio 2021. 
aunque al no existir datos sobre la comunidad GLBTIQ+ y la profesión es importante nombrarla a manera de desafío para futuras investigaciones.

Con estas ideas delimitadas, el análisis se dividirá bajo este marco conceptual aplicado a las periodistas, a nivel mundial y con especificidades de Ecuador. La primera parte, con base a la discriminación, se establecerá limitaciones y prejuicios que evitan el desarrollo profesional en el medio de comunicación por el hecho de ser mujer, con base en los llamados techo de cristal y suelo pegajoso; $\mathrm{y}$, la segunda, versará sobre las violencias que afronta una mujer que ejerce el periodismo.

Definido por Morrison et al. (1994), el techo de cristal es aquella barrera invisible que impide que mujeres calificadas puedan ocupar cargos en alta dirección o de responsabilidad; y, para el caso de los medios de comunicación, hay evidencias de los límites para que ellas puedan participar de ciertas tareas, además de restricciones para progresar (UNESCO y Universidad de Oxford, 2018). Dado que esto se abordó en el apartado anterior, se sumarán actitudes recurrentes sobre discriminación por temas etarios y de voces autorizadas; no se realizará un abordaje interseccional sobre aspectos étnicos por considerar que esas realidades requieren una extensa atención.

Como figura en el Informe Regional del Proyecto de Monitoreo Global de Medios (WACC et al., 2015), se mantiene y refuerza el estereotipo occidental de belleza, que incluye la edad de las mujeres como algo relevante para escoger presentadoras o reporteras; tal es así que, en estos puestos un $43 \%$ de ellas tienen de 19 a 34 años, mientras que los hombres en el mismo rango de edad representan apenas el 14\%, frente a un 53\% que tienen entre 35 y 49 años, y $26 \%$ están entre los 50 y 64. Queda claro que las periodistas se valoran por su juventud, al contrario de sus pares donde su experiencia prima.

Sobre la cuestión de voces autorizadas, como parte del Informe de la Relatoría para la Libertad de Expresión de la CIDH (OEA, 2018), se especifica que la discriminación, también, "se traduce en la escasa participación de las mujeres en la agenda de los medios de comunicación" (p. 67); por ejemplo, en el rol de experta o comentarista, según el Proyecto de Monitoreo Global de Medios regional (WACC et al., 2015), se cuantificó que las funciones de ellas son reducidas: un $32 \%$ en política y gobierno, $15 \%$ en activismo y causas sociales, $13 \%$ en la academia y $11 \%$ en funciones públicas. Cabe señalar que esto no tiene que ver con las capacidades y formación, sino la poca visibilización de periodistas o interlocutoras especializadas.

Relacionado también con la discriminación está el denominado suelo pegajoso que, para investigadoras como Chavero (2020), es aquel del que se habla menos, pero es el que más acaba con las profesionales en medios de comunicación. Son las dificultades que impiden conciliar el trabajo doméstico, al estar mayormente delegado a las mujeres; esto ocasiona que funcione como una especie de pegamento al piso para despegar en su desarrollo. Sin ahondar en el asunto, por ser un factor común al momento de seleccionar personal o profesión (Ragazzo, 2018), se hace evidente, por ejemplo, cuando en las entrevistas para ingresar a un 
trabajo o formación a casi a todas las mujeres se les pregunta sobre aspectos relacionados a la maternidad y el matrimonio, cosa que rara vez les ocurre a los hombres.

Sobre las violencias contra las mujeres se puede afirmar que es una preocupación mundial porque impide y anula el ejercicio de sus derechos (OEA, 1994). Los actos contra las periodistas no son aislados, sino un problema estructural (OEA, 2018) que se reproduce a nivel nacional e internacional.

Por la cantidad de material encontrado sobre hechos que incluyen al Ecuador, en la Tabla 1 se sistematizan algunos que dan cuenta de esta realidad. Con este breve ejercicio, se puede notar que, con las nuevas tecnologías, prima la violencia en línea sin desmerecer la existencia de otras herramientas para acosar, amenazar e incluso llegar al plano sexual o la muerte.

Esta revisión de las violencias y discriminaciones, junto a las pruebas fehacientes de su presencia en Ecuador y en el mundo, es una invitación a leer la totalidad de los documentos citados junto a otros tantos de circulación libre; el objetivo es poner en el debate esta problemática que requiere acciones especializadas de los Estados y los medios de comunicación.

Así también, involucrar a las audiencias como una contraparte que garantice la seguridad de las mujeres y su derecho a ejercer el periodismo como profesión por evidenciar que son fuente del problema y, por tanto, de posibles soluciones.

\section{Método}

Para el análisis cuantitativo se utilizará como fuente el Registro Público de Medios del Consejo de Comunicación, con corte al 22 de noviembre de 2020, donde constan 818 medios entre radios, impresos, televisoras, sistemas AVS y digitales. De ellos, se utilizará la información de los colaboradores que suman 12,893 empleados; particularmente, 6,147 están vinculados al área de comunicación. Cabe resaltar que este catastro es obligatorio, según la norma vigente que reza:

Art. 88.- Registro público.- Los medios de comunicación social se registrarán obligatoriamente en un catastro a cargo del Consejo de Regulación, Desarrollo y Promoción de la Información y Comunicación dicho catastro deberá contener datos generales que se determinarán en el reglamento.

Este registro no constituye una autorización para el funcionamiento del medio de comunicación.

Los medios de comunicación que no cumplan con la obligación de registro no podrán pautar publicidad de ninguna entidad del Estado. (Ley Orgánica Reformatoria a la Ley Orgánica de Comunicación, 2019). 


\section{Tabla 1}

Violencias y acciones violentas contra las periodistas a nivel mundial

\begin{tabular}{|c|c|}
\hline $\begin{array}{c}\text { Violencias o } \\
\text { acciones violentas } \\
\end{array}$ & Datos \\
\hline $\begin{array}{l}\text { - Acoso sexual } \\
\text { - Acoso en línea } \\
\text { - Violencia sexual } \\
\text { - Amenazas }\end{array}$ & $\begin{array}{l}\text { "La proporción de mujeres entre las víctimas mortales también ha aumentado y las } \\
\text { periodistas se enfrentan a un aumento de las agresiones por motivos de género, como el } \\
\text { acoso sexual (incluso en línea), la violencia sexual y las amenazas de violencia" } \\
\text { (Organización de las Naciones Unidas [ONU], 2019, p. 3). }\end{array}$ \\
\hline $\begin{array}{l}\text { - Asesinato, } \\
\text { - Violencia sexual } \\
\text { - Acoso sexual } \\
\text { - Intimidación } \\
\text { - Abuso de poder } \\
\text { - Amenaza }\end{array}$ & $\begin{array}{l}\text { "Esta violencia se manifiesta de distintas formas, desde el asesinato, la violencia sexual, } \\
\text { incluido el acoso sexual hasta la intimidación, abuso de poder y amenazas basadas en el } \\
\text { género. De acuerdo con la información disponible, la violencia contra las mujeres es } \\
\text { perpetrada por distintos actores, como funcionarios del Estado, fuentes de información o } \\
\text { colegas y tiene lugar en diversos contextos y espacios, incluyendo la calle, el lugar de } \\
\text { trabajo y las oficinas o instituciones estatales" (OEA, 2013, p. 474). }\end{array}$ \\
\hline $\begin{array}{l}\text { - Violencia sexual } \\
\text { - Abuso sexual } \\
\text { - Violación sexual }\end{array}$ & $\begin{array}{l}\text { "38. [...] los ataques documentados adoptaron tres formas diferenciadas: violación sexual } \\
\text { contra periodistas en represalia por su trabajo, abuso sexual de periodistas en cautiverio } \\
\text { o bajo detención, y violencia sexual por parte de las turbas contra periodistas que cubren } \\
\text { actos públicos" (OEA, 2018, pág. 27). }\end{array}$ \\
\hline - Acoso en línea & $\begin{array}{l}\text { "El acoso online en redes sociales y sitios web se dirige a mujeres periodistas de cualquier } \\
\text { origen político, religioso y étnico. Uno de los aspectos principales de estos ataques es que } \\
\text { son de género y suelen estar sexualizados. Una encuesta de la FIP realizada en } 2018 \\
\text { mostró que el } 43 \% \text { de las mujeres encuestadas habían sido sometidas al acoso online" } \\
\text { (Federación Internacional de Periodistas [FIP], 2019, p. 1). }\end{array}$ \\
\hline - Ataques sexuales & $\begin{array}{l}\text { "Pocos casos de ataques sexuales contra periodistas han sido documentados a raíz de } \\
\text { fuertes estigmas culturales y profesionales. Pero ahora decenas de periodistas se animan } \\
\text { a revelar que han sido víctimas de abusos sexuales mientas cumplían con su labor } \\
\text { informativa" (Comité para la Protección de los Periodistas [CPJ], 2011, párr. 1). }\end{array}$ \\
\hline $\begin{array}{l}\text { - Acoso en línea } \\
\text { - Ataques } \\
\text { - Amenaza de } \\
\text { muerte } \\
\text { - Acoso } \\
\text { - Amenaza }\end{array}$ & $\begin{array}{l}\text { De } 597 \text { periodistas y trabajadoras en medios de comunicación que fueron encuestadas: el } \\
63 \% \text { señaló que había sido amenazado o acosado en línea; el } 58 \% \text { indicó que había sido } \\
\text { amenazado o acosado en persona; y, el } 26 \% \text { dijo que habían sido atacados físicamente. } 1 \\
\text { de cada } 10 \text { dijo haber sido amenazada de muerte; } 70 \% \text { de las encuestadas han } \\
\text { experimentado alguna forma de acoso, amenaza o ataque en los últimos } 5 \text { años; un tercio } \\
\text { de las encuestadas considera retirarse de la profesión luego de recibir algún tipo de ataque } \\
\text { (Ferrier, M. 2018). }\end{array}$ \\
\hline $\begin{array}{l}\text { - Violencia en } \\
\text { línea }\end{array}$ & $\begin{array}{l}\text { "Un análisis de más de dos millones de tuits realizado por el think tank Demos revela que } \\
\text { las mujeres periodistas recibían aproximadamente tres veces más comentarios abusivos } \\
\text { que los hombres en Twitter" (Demos, 2014). }\end{array}$ \\
\hline $\begin{array}{l}\text { - Violencia en } \\
\text { línea } \\
\text { - Violencia física } \\
\text { - Acoso } \\
\text { - Violencia sexual }\end{array}$ & $\begin{array}{l}\text { Además de los riesgos y amenazas que experimentan sus homólogos masculinos, también } \\
\text { están expuestos al acoso y la violencia de género en el campo y en la sala de redacción. } \\
\text { Estos ataques pueden ser de naturaleza física o virtual y, con frecuencia, se sexualizan } \\
\text { (Posetti y Storm, 2018). }\end{array}$ \\
\hline
\end{tabular}

Fuente: Documentos citados.

Se complementarán estos datos con el Monitoreo de Contenidos del Consejo de Comunicación (Consejo de Comunicación, 2020a), con una investigación específica donde se realizó un análisis de la incorporación del enfoque de género.

Global Media Journal México, 18(34), 94-110, enero - junio 2021. 
La muestra corresponde a 24 medios de comunicación monitoreados en junio de 2020. Fueron nueve televisoras: Gamavisión, Televicentro, RTS, RTU, TC Televisión, Teleamazonas, Ecuador TV, Ecuavisa y Canal Uno; ocho radios: Radio Centro, Visión, Pichincha Universal, La Red, Radio Tricolor, Platinum, Excelencia Radio y FB Radio; y, siete impresos: Extra, Expreso, La Hora, Últimas Noticias, El Comercio, El Universo y El Telégrafo.

\section{Resultados}

\section{La presencia de las mujeres en los medios de comunicación en Ecuador}

Con una conciencia básica tanto del marco legal, como de los desafíos y obstáculos que tienen las mujeres al laborar en medios de comunicación, a continuación se ofrecerán algunas cifras relacionadas al caso ecuatoriano a través de dos herramientas del
Consejo de Comunicación: el Registro Público de Medios (RPM) (Consejo de Comunicación, 2020b) y el Monitoreo de Medios (Consejo de Comunicación, 2020a).

Con base en el RPM (Consejo de Comunicación, 2020b) a corte del 22 de noviembre de 2020, son 818 medios de comunicación registrados entre radios, impresos, televisoras, sistemas AVS y digitales. En conjunto suman 12,893 empleados, de los cuales 6,147 están vinculados al área de comunicación directamente: 1,967 mujeres, quienes son el 32\% y, 4,207 hombres, quienes son el $68 \%$. En la Figura 1 se grafica la diferencia en la participación de los empleados vinculados a comunicación.

Para el caso de las 62 televisoras registradas, en el área de comunicación, la cantidad de hombres representa más del doble de mujeres empleadas. En la Tabla 2 se muestra que la diferencia se mantiene tanto en comunitarios, privados y públicos; incluso, en los dos últimos se llega a triplicar la presencia de los hombres en comparación con las mujeres.

\section{Figura 1}

Número de empleados inscritos en el Registro Público de Medios
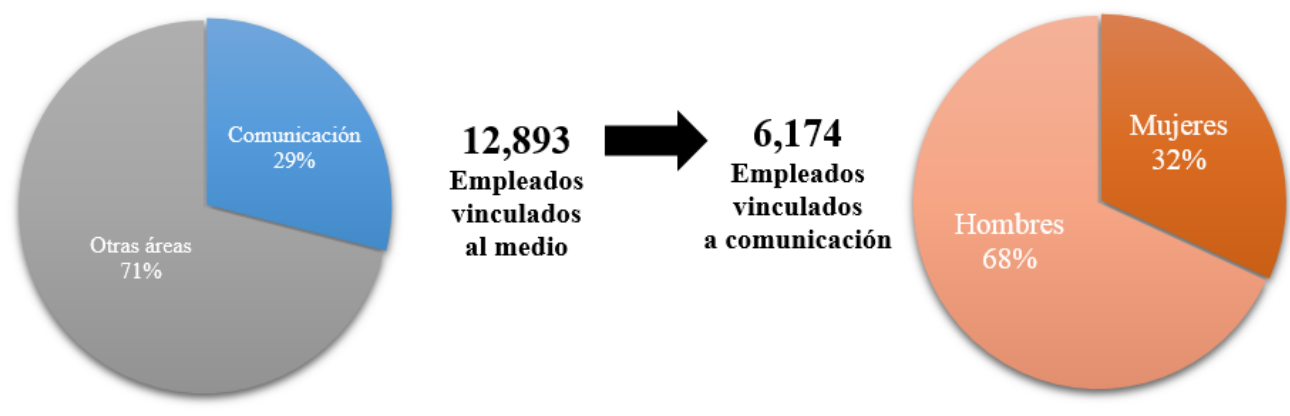

Fuente: Registro Público de Medios (Consejo de Comunicación, 2020b).

Global Media Journal México, 18(34), 94-110, enero - junio 2021. 
Tabla 2

Número de empleados en televisión inscritos en el Registro Público de Medios

\begin{tabular}{lcccc}
\hline \multicolumn{1}{c}{ Tipo } & $\begin{array}{c}\text { Total de } \\
\text { empleados }\end{array}$ & $\begin{array}{c}\text { Empleados en } \\
\text { comunicación }\end{array}$ & Mujeres & Hombres \\
\hline Comunitario & 41 & 32 & 11 & 21 \\
Privado & 1,729 & 1,275 & 327 & 948 \\
Público & 765 & 309 & 77 & 232 \\
Total & 2,535 & 1,616 & 415 & 1,201 \\
\hline Fuente: &
\end{tabular}

Fuente: Registro Público de Medios (Consejo de Comunicación, 2020b).

En el caso de las 498 radios registradas, la cantidad de hombres en el área de comunicación es más del doble que las mujeres, en los tres tipos de medio. En la Tabla 3 se muestra la distribución.

De los 162 impresos que son periódicos, diarios, revistas y formatos relacionados, la diferencia es casi de dos a uno entre hombres y mujeres. Cabe señalar, que en el ámbito privado es donde hay un mayor número de mujeres, sin que por ello signifique que se haya alcanzado ni de cerca un equilibrio. En la Tabla 4 se muestra esta distribución.

\section{Tabla 3}

Número de empleados en radio inscritos en el Registro Público de Medios

\begin{tabular}{lcccc}
\hline \multicolumn{1}{c}{ Tipo } & $\begin{array}{c}\text { Total de } \\
\text { empleados }\end{array}$ & $\begin{array}{c}\text { Empleados en } \\
\text { Comunicación }\end{array}$ & Mujeres & Hombres \\
\hline Comunitario & 211 & 198 & 57 & 141 \\
Privado & 2,345 & 1,912 & 537 & 1,375 \\
Público & 735 & 134 & 39 & 95 \\
Total & 2,391 & 2,244 & 633 & 1,611 \\
\hline Fuente: Registro Público de Medios (Consejo de Comunicación, 2020b).
\end{tabular}

Fuente: Registro Público de Medios (Consejo de Comunicación, 2020b).

\section{Tabla 4}

Número de empleados en impresos inscritos en el Registro Público de Medios

\begin{tabular}{ccccc}
\hline Tipo & $\begin{array}{c}\text { Total de } \\
\text { empleados }\end{array}$ & $\begin{array}{c}\text { Empleados en } \\
\text { comunicación }\end{array}$ & Mujeres & Hombres \\
\hline Comunitario & 10 & 7 & 3 & 4 \\
Privado & 5,209 & 1,773 & 732 & 1,041 \\
Público & 1,258 & 183 & 53 & 130 \\
Total & 6,477 & 1,963 & 788 & 1,175 \\
\hline
\end{tabular}

Fuente: Registro Público de Medios (Consejo de Comunicación, 2020b). 
Para una mejor visualización en lo que ocurre en los tres tipos de medios, en el Gráfico 2 se representa este desequilibrio, que llega a más de dos hombres por cada mujer en el área de comunicación. De los
5,823 empleados que corresponden a la suma de radio, televisión, y medios impresos 1,836 son mujeres y 3,987 son hombres.

\section{Gráfico 2}

Número de empleados por tipo de medio inscritos en el Registro Público de Medios
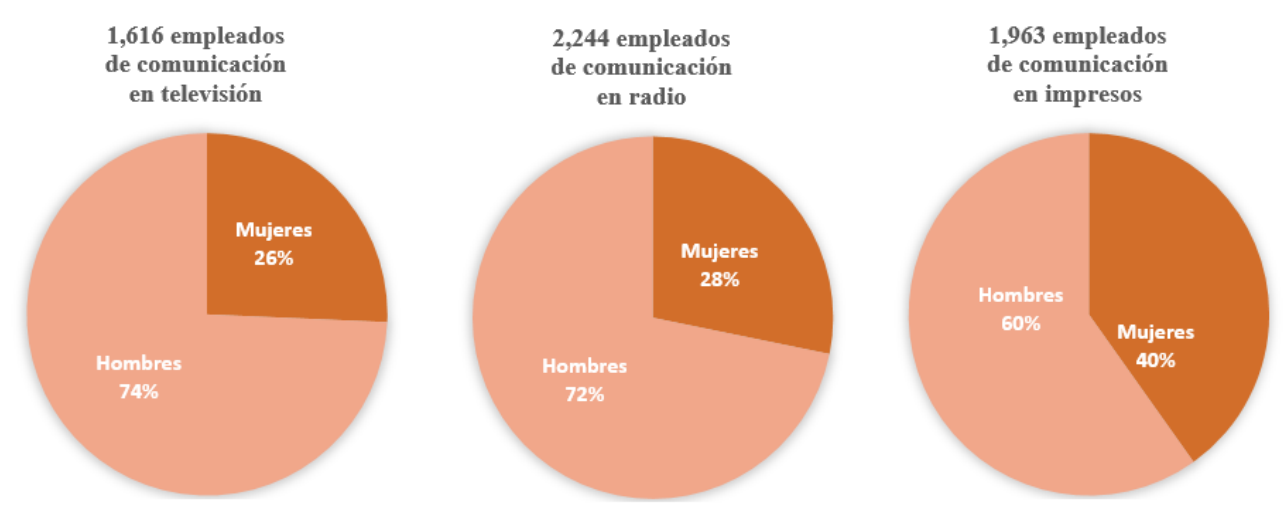

Fuente: Registro Público de Medios (Consejo de Comunicación, 2020b).

Cabe señalar que el Registro Público de Medios (Consejo de Comunicación, 2020b) es un catastro obligatorio para los medios de comunicación, conforme el artículo 88 de la Ley de Comunicación reformada (2019); sin embargo, no representa el universo total de los medios de comunicación existentes en Ecuador. Aun así, es un número considerable para evaluar de forma técnica la participación de las mujeres en la esfera mediática y cuantificar los avances en temas de derechos.

Es evidente que se mantiene el proceso de aniquilación simbólica, mismo que no pasa por un tema de profesionalización, como lo demostraron las investigaciones expuestas previamente, sino de inserción de las mujeres en el ámbito laboral de los medios $\mathrm{y}$, sobre todo en ciertas áreas.
Ahora bien, para contrastar con realidad hacia el público se utilizará el Monitoreo de Contenidos del Consejo de Comunicación (2020a), con una investigación específica donde se realizó un análisis de la incorporación del enfoque de género.

La muestra corresponde a 24 medios de comunicación, monitoreados en junio de 2020. Fueron nueve televisoras: Gamavisión, Televicentro, RTS, RTU, TC Televisión, Teleamazonas, Ecuador TV, Ecuavisa y Canal Uno; ocho radios: Radio Centro, Visión, Pichincha Universal, La Red, Radio Tricolor, Platinum, Excelencia Radio y FB Radio; y, siete medios impresos: Extra, Expreso, La Hora, Últimas Noticias, El Comercio, El Universo y El Telégrafo.

Los resultados sobre la participación en televisión, en los 28 programas informativos 
analizados reporta que, del total de personas que cumplen algún rol en el espacio relacionadas a contenidos: 173 son hombres y 164 son mujeres. Eso representa un ponderado donde de cada 100 de profesionales en esta área, la mitad más uno son hombres. En particular, en el rol de periodista de campo de cada 100 personas, 52 serían hombres y 48 mujeres.

En el caso de la radio, de los 15 programas monitoreados: 37 son hombres y 18 son mujeres; y, en ponderación, de cada 100 personas que cumplen diferentes roles 67 son hombres y 33 mujeres. Cabe señalar que, el rol de periodista de campo es el único en el que se encontró equilibrio de 1 a 1 en este tipo de medio.

Por su parte, de los siete impresos analizados: 378 son hombres y 160 son mujeres. La ponderación evidencia que de cada 100 personas, 70 corresponde a hombres y 30 a mujeres, es decir, hay una diferencia de tres a uno.

Los datos expuestos, visto de manera general, ofrecen una muestra técnica de la inclusión del enfoque de género en los contenidos de los medios de comunicación ecuatorianos con base a la presencia de mujeres y hombres; estos últimos preferidos mayormente para presentarlos hacia el público.

Se puede afirmar que persisten ciertas estructuras históricas en los medios de comunicación ecuatorianos, sin desestimar que se ha pasado de la exclusión total o el anonimato de las mujeres a participar con voz en ciertos ámbitos que la tradición destinaba para los hombres.

\section{Conclusiones y recomendaciones}

Si bien se reconoce la necesidad de protección a periodistas y trabajadores de la comunicación en general, "[...] a la voluntad de silenciamiento que caracteriza a los ataques contra la prensa, se agregan estigmatizaciones propias de la violencia que se sustenta en construcciones estereotipadas de género, asignación de roles y reproducen modelos estructurales [...]" (Consejo de Comunicación, 2019, pág. 221), mismas que merecen acciones de prevención y erradicación especializada, es decir, incluir en todos los procesos la transversalidad de género, que es:

Art. 8., d) [...] Incorporación de los diversos enfoques de los derechos de igualdad de género establecidos en la Constitución, instrumentos internacionales y leyes, a todo nivel y en todo el ciclo de la gestión pública, y privada y de la sociedad, en general a fin de garantizar un tratamiento integral de esta problemática (Ley para Prevenir y Erradicar la Violencia contra la Mujer, 2018).

Por ello, con base a instrumentos de la Relatoría Especial para la Libertad de Expresión de la CIDH (OEA, 2018), el Informe del Secretario de la ONU (ONU, 2019), la Declaración y Plataforma de Acción de Beijing (ONU Mujeres, 1995), la UNESCO (2014), ONU Mujeres (2016), y otros documentos socializados en este artículo, se sugiere propender a

Global Media Journal México, 18(34), 94-110, enero - junio 2021. 
la "igualdad sustantiva” (ONU Mujeres, 2016, pág. 592) en tres esferas interrelacionadas: primera, la corrección de la desventaja socioeconómica de las mujeres; segunda, la lucha contra las violencias y discriminaciones; y, tercera, con el fortalecimiento del poder de acción, la voz y la participación de las mujeres. Dichas esferas aplicadas en el ámbito comunicacional con acciones como:

- Reconocer esta problemática especializada en el área de comunicación con la generación de marcos legales y otros documentos que la visibilicen; así como, sanciones efectivas y oportunas para para los agresores.

- Generar programas de protección para periodistas que incluyan la urgente acción sobre las violencias y discriminaciones por temas de género. Esta responsabilidad es compartida tanto por Estado como por las instituciones mediáticas.

- Campañas de sensibilización sobre violencias y discriminaciones al interior de los medios de comunicación; al tiempo, que su implementación se refleje en la calidad en los contenidos.

- Elaborar guías especializadas de autoprotección y entrenamiento sobre seguridad y género para las mujeres periodistas.

- Elaborar investigaciones cuantitativas y cualitativas sobre periodismos y género, que permitan identificar problemas y posibles soluciones.
- Combatir los estereotipos de género en relación con los roles, sobre todo aquellos relacionados con generación de contenidos. Con ello se espera, entre otros resultados, seleccionar el personal de pantalla y tras cámara con base en sus capacidades.

- Incentivar el empoderamiento y la organización de las mujeres como profesionales para generar redes de apoyo y seguimiento.

- Fortalecer los procesos de profesionalización de las mujeres en el ámbito comunicacional, de la mano con los procesos de inserción en el mercado laboral.

- No basta con equiparar cifras de participación, es importante trabajar con los hombres que laboran en medios de comunicación para fortalecer procesos complementarios como el desarrollo de nuevas masculinidades.

A manera de reflexión final, con todo lo expuesto, se puede decir que los hechos hablan de un problema estructural histórico de violencias y discriminación contra las periodistas y trabajadoras de la comunicación. La existencia y cumplimiento de los derechos de las mujeres no se puede darse por sentado porque los avances en la visibilización y representación son resultado de sus luchas permanentes en todos los niveles.

No es tan lejana la historia del anonimato y exclusión de las mujeres en medios de comunicación y profesionalización, por lo que las acciones para superar esta problemática deben ser articuladas entre 
el Estado, las instituciones mediáticas, las audiencias

y cualquier actor social que pueda aportar a la prevención y erradicación de todo aquello que impide se desarrollen laboralmente. Esto, como un derecho individual de aquellas que forman parte de la mitad de la población del mundo; y, como una obligación general para garantizar un real ejercicio de la libertad de expresión y de prensa.

\section{Referencias bibliográficas}

Beate, J., Folker, H., Oller, M., Shapiro, I., Kenneth, A., de Beer, A., Hoxha, A., Moreira, S. V., Rafter, K., Skjerdal, T., Splendore, S., y Tandoc Jr., E. C. (2019). Profiles on journalists: Demographic and employment patterns. En T. Hanitzsch, F. Hanusch, J. Ramaprasad y A. S. de Beer (Eds.), Worlds of journalism. Journalistic cultures around the blobe (pp. 84-119). Columbia University Press.

Calvopiña, V. (22 de mayo de 2019). Zoila Ugarte: un periodismo por ellas y para ellas. Wambra Radio. https://bit.ly/3hj9OnL

Casa de la Cultura Ecuatoriana. (s.f.). Zoila Ugarte de Landivar. CCE. https://bit.ly/3jTn2Jk

Chavero, P. (14 de octubre de 2020). La mujer en los medios: de la invisibilización a la proclama. En Conversatorio Virtual: Mujeres, libertad de expresión y medios de comunicación Consejo de $\begin{array}{lllll}\text { Comunicación. } & \text { [Publicación } & \text { Facebook video] }\end{array}$ https://www.facebook.com/ConsejoComEc/videos/791068481713162

Comité para la Protección de los Periodistas [CPJ]. (07 de junio de 2011). El Crimen silenciado: Violencia sexual y periodistas. https://bit.ly/3dO0B4B

Consejo de Comunicación. (2017). Discriminación hacia las mujeres y su representación en medios de comunicación.

Consejo de Comunicación. (2019). Propuesta de Principios Generales para la Protección a Trabajadores de la Comunicación. Enfoques de la Comunicación 3. Protección a Periodistas y Trabajadores de la Comunicación, 216-223. https://bit.ly/3hoDobm

Consejo de Comunicación. (2020a, junio). Componente cualitativo y jurídico al informe $N^{\circ}$ CRDPIC-CGDICDTMC-2020-002-INF correspondiente al monitoreo del enfoque de género en los medios de comunicación social.

Consejo de Comunicación. (2020b). Registro Público de Medios. https://bit.ly/3xtfdhJ

Constitución de la República del Ecuador. (20 de octubre de 2008). https://bit.ly/2VcBRN2

Convención sobre la Eliminación de Todas las Formas de Discriminación contra la Mujer. (18 de diciembre de 1979). ONU. https://bit.ly/3ysqqPG

Declaración de Chapultepec. (1994). https://bit.ly/3xlROhV

Global Media Journal México, 18(34), 94-110, enero - junio 2021. 
Demos. (24 de Agosto de 2014). Demos: Male celebrities receive more abuse on Twitter than women. https://bit.ly/3hJy55w

Federación Internacional de Periodistas [FIP]. (2019). Guía para sindicatos y medios de comunicación para combatir el acoso online a mujeres periodistas. https://bit.ly/3jLkD3r

Ferrier, M. (2018). Attacks and Harassment: The impact on female journalists and their reporting. The News Media (and) Development Network. https://bit.ly/2STcNdc

Hanitzsch, T. (2007). Deconstructing journalism culture: Toward a universal theory. Mass Communication Theory, 17(4), 367-385. https://doi.org/10.1111/j.1468-2885.2007.00303.x

International Women's Media Foundation [IWMF]. (2011). The Global Report on the Status of Women in the News Media. https://bit.ly/3xtfFfV

Ley Orgánica Reformatoria a la Ley Orgánica de Comunicación. (2019). Ecuador. https://bit.ly/3jXIEog

Ley para Prevenir y Erradicar la Violencia contra la Mujer. (31 de enero de 2018). Ecuador. https://bit.ly/3ys6WuA

Mead, M. (1982). Sexo y temperamento: en tres sociedades primitivas. Paidós.

Morrison, A., White, R., y Van Velsor, E. (1994). Breaking the Glass Ceiling: Can Women Reach the Top of America's Largest Corporations. Perseus Books.

Oller, M., y Chavero, P. (2018). Journalism in Latin America: Journalistic culture of Ecuador. Editorial Académica Española.

Oller, M., Chavero, P., Carrillo, J., y Cevallos, P. (2016). ¿Determina el género la percepción del rol profesional de1@s periodistas en Ecuador? Razón y Palabra, 20(93), 229-250. https://bit.ly/3jZsE5d

ONU Mujeres. (1995). Declaración y Plataforma de Acción de Beijing. https://bit.ly/3xfjhlv

ONU Mujeres. (2016). El progreso de las mujeres en el mundo 2015-2016. Transformar las economías para realizar los derechos. Resumen. Revista Estudos Feministas, 24(2), 589-614. https://bit.ly/3jZGk03

ONU Mujeres. (25 de febrero de 2020). Visualizar los datos: La representación de las mujeres en la sociedad. https://bit.ly/3qOc6y6

Organización de las Naciones Unidas [ONU]. (16 de agosto de 2019). La seguridad de los periodistas y la cuestión de la impunidad. Informe del Secretario General. https://bit.ly/36duO99

Organización de los Estados Americanos [OEA]. (1994). Convención Belém do Pará. https://bit.ly/3AtOeV3

Organización de los Estados Americanos [OEA]. (2013). Informe Anual de la Relatoría Especial para la Libertad de Expresión de la Comisión Interamericana de los Derechos Humanos 2013. https://bit.ly/3xlN9Nc

Organización de los Estados Americanos [OEA]. (2018). Mujeres periodistas y libertad de expresión. Discriminación y violencia basada en el género contra las mujeres periodistas por el ejercicio de su profesión. Relatoría Especial para la Libertad de Expresión de la Comisión Interamericana de Derechos Humanos. https://bit.ly/2SRKziY

Paladines, C. (2004). Erophilia. Conjeturas sobre Manuela Espejo. Ediciones Abya-Yala.

Global Media Journal México, 18(34), 94-110, enero - junio 2021. 
Plaza, M. (2014). Imagen y representación social acerca de la mujer periodista en las teleaudiencias. Estudio de los noticieros estelares de Ecuavisa y Teleamazonas [tesis de licenciatura, Pontificia Universidad Católica del Ecuador]. Repositorio Institucional. https://bit.ly/3dKXA5d

Posetti, J., y Storm, H. (2018). Violence Against Women Journalists -Online and Offline. En UNESCO, Setting the gender agenda for communication policity (pp. 75-92). https://bit.ly/3xkRFeR

Proyecto de Monitoreo Global de Medios. (2015a). ¿Quién figura en las noticias? https://bit.ly/3i1BFb2

Proyecto de Monitoreo Global de Medios. (2015b). ¿Quién figura en las noticias? América Latina?. https://bit.ly/3dX3zDZ

Ragazzo, M. (2018). La persistencia de la cultura androcéntrica al interior de las organizaciones. En M. Cano Flores, O. González Muñoz y V. Velázquez Romero (Coords.), Reflexiones sobre el desarrollo empresarial (pp. 57-71). https://bit.ly/3hl5cNG

Rodas, R. (2011). Zoila Ugarte de Landivar. Patriota y Republicana “Heroína ejemplar del feminismo”. Comisión de Transición. http://repositorio.iaen.edu.ec/handle/24000/4440

Ross, K., Boyle, K., Carter, C., y Ging, D. (2016). Women, men and news: it's life, Jim, but not as we know it. Journalism Studies, 19(6), 824-845. https://doi.org/10.1080/1461670X.2016.1222884

Tuchman, G., Daniels, A., y, Benoit, J. (1978). Hearth and home: Images of women in the mass media. Oxford University Press.

UNESCO. (2014). Indicadores de Género para los Medios de Comunicación. Marco de Indicadores para Evaluar la Sensibilidad en Materia de Género en las Operaciones y Contenidos Mediáticos. https://bit.ly/3hwfA44

UNESCO, y Universidad de Oxford. (2018). Tendencias mundiales en libertad de expresión y desarrollo de los medios. Informe Mundial 2017/2018. https://bit.ly/3hGu4OW

WACC, Funadación Gamma, Media Monitoring Africa, Code for South Africa, ONU Mujeres, y UNESCO. (2015). Proyecto de Monitoreo Global de Medios. Informe Regional. https://bit.ly/3wrQyJ9

Global Media Journal México, 18(34), 94-110, enero - junio 2021. 